Supporting information

\title{
Direct observation of amide bond formation in a plasmonic nanocavity triggered by single nanoparticle collisions
}

Na Kong, ${ }^{\dagger, \ddagger}$ Jing Guo, ${ }^{\ddagger}$ Shuai Chang, ${ }^{\S}$ Jie Pan, ${ }^{\ddagger}$ Jianmei Wang, ${ }^{\dagger}$ Jianghao Zhou, ${ }^{\ddagger}, \S$ Jing Liu, ${ }^{\ddagger}$ Hong Zhou, ${ }^{\dagger \#}$ Frederick M. Pfeffer,${ }^{\dagger}$ Jingquan Liu, ${ }^{\xi}$ Colin J. Barrow, ${ }^{\dagger}$ Jin He, ${ }^{\star,, \neq, l}$ and Wenrong Yang ${ }^{*} \dagger$

†School of Life and Environmental Sciences, Deakin University, Geelong, VIC 3216, Australia

‡Department of Physics, Florida International University, Miami, FL 33199, USA

\$The State Key Laboratory of Refractories and Metallurgy, the Institute of Advanced Materials and Nanotechnology, Wuhan University of Science and Technology, Wuhan 430081, China

"Shandong Province Key Laboratory of Detection Technology for Tumor Markers, Linyi University, Linyi, Shandong 276005, P. R. China

${ }^{\xi}$ College of Material Science and Engineering, Institute for Graphene Applied Technology Innovation, Qingdao University, Qingdao 266071, China

"Biomolecular Science Institute, Florida International University, Miami, FL 33199, USA

*E-mail: wenrong.yang@deakin.edu.au; jinhe@fiu.edu 


\section{Chemicals and reagents}

Cysteamine (CA), 3-Mercaptopropionic acid (MPA), 3,3'-Dithiodipropionic acid di(Nhydroxysuccinimide ester) (Lomant), high density polyethylene (HDPE), Potassium ferrocyanide (98.5\% purity, analysis grade) were purchased from Sigma-Aldrich. Phosphate Buffered Saline (PBS) powder and absolute Ethanol (200 proof) were purchased from Fisher Scientific. All the chemicals were used directly without further purification. All the aqueous solutions were prepared using deionized (DI) water $(\sim 18$ M Ohm).

\section{The preparation and characterization of Gold nanoelectrodes (GNE)}

A two-stage electrochemical etching method generally used for scanning tunnelling microscope (STM) tip was utilized to fabricate GNE. ${ }^{1-2}$ In the first step, the tip of a 10 mm long gold wire $(0.25 \mathrm{~mm}, 99.998 \%)$ was dipped into the etching solution that mixed by ethanol and hydrochloric acid $(\mathrm{v}: \mathrm{v}=1: 1)$. A Pt wire of circular shape surrounding the gold wire was served as the other electrode. The applied AC voltage has a frequency of $4.2 \mathrm{kHz}$ and amplitude of $25.5 \mathrm{~V}$. In the second "fine-etching" stage, the tip was continued sharpened with AC voltage of about half the previous magnitude. After the electrochemical etching process, the tip was cleaned in piranha acid (warning, piranha etch can react explosively with organics) for $10 \mathrm{~min}$, sequentially rinsed by DI water and ethanol, then dried in an Argon flow. The prepared tip was insulated with high density polyethylene (HDPE) to afford the final GNE.

Figure S1a shows a typical SEM image of a prepared GNE. A HDPE insulating layer covers most of the GNE tip surface but leaving the apex exposed. Figure S1b shows the optical image of a GNE during the SERS measurement, with the laser beam focused on the GNE apex.

Figure S1c shows the cyclic voltammograms (CVs) of a GNE, which were measured at $50 \mathrm{mV} / \mathrm{s}$ in $1 \mathrm{M} \mathrm{KCl}$ solution containing $100 \mathrm{mM}$ Ferrocyanide ions. The $\mathrm{CV}$ of the GNE before modification (black colour) showed the typical sigmoidal shape for a ultrasmall electrode. Based on the diffusion-limited current $I_{d}$ in the $C V$, we can estimate the exposed surface area of GNE apex using the following equation: ${ }^{3}$

$i_{d}=m F D C \sqrt{2 \pi A_{e f f}}$. 
The $\mathrm{A}_{\mathrm{eff}}$ is the effective electrode area, $\mathrm{m}$ is a geometry factor, $\mathrm{F}$ is the Faraday constant, $\mathrm{D}$ and $\mathrm{C}$ are the diffusion constant and concentration of ferrocyanide ions, respectively. The geometry factor $\mathrm{m}=1$ if the electrode is hemispherical. For other geometries, such as inlaid disc, sphere cap and hemispheroid, m changes slightly (normally less than $10 \%$ ). For example, $\mathrm{m}=1.1$ if the aspect ratio of an oblate hemispheroid is 6 . Therefore, we used $m=1$ here for the estimation of the effective exposed area. For CV curve (black color) shown in Figure $\mathrm{S} 1 \mathrm{C}$, the $\mathrm{I}_{\mathrm{d}}=23 \mathrm{nA}$. Therefore, $\mathrm{A}_{\mathrm{eff}}$ of GNE was estimated about $1.65 \mu \mathrm{m}^{2}$. The effective radius of the exposed GNE radius is thus calculated to be $512 \mathrm{~nm}$ assuming a hemispherical shape. In general, the measured $i_{d}$ values are in the range of $10 \mathrm{nA}$ to $40 \mathrm{nA}$, which means that the effective surface area of GNE is in the range from $0.32 \mu \mathrm{m}^{2}$ to $5.2 \mu \mathrm{m}^{2}$. After CA modification, $\mathrm{I}_{\mathrm{d}}$ of CAGNE (blue CV) is reduced $61.2 \%$, suggesting the surface modification of CA. ${ }^{4}$

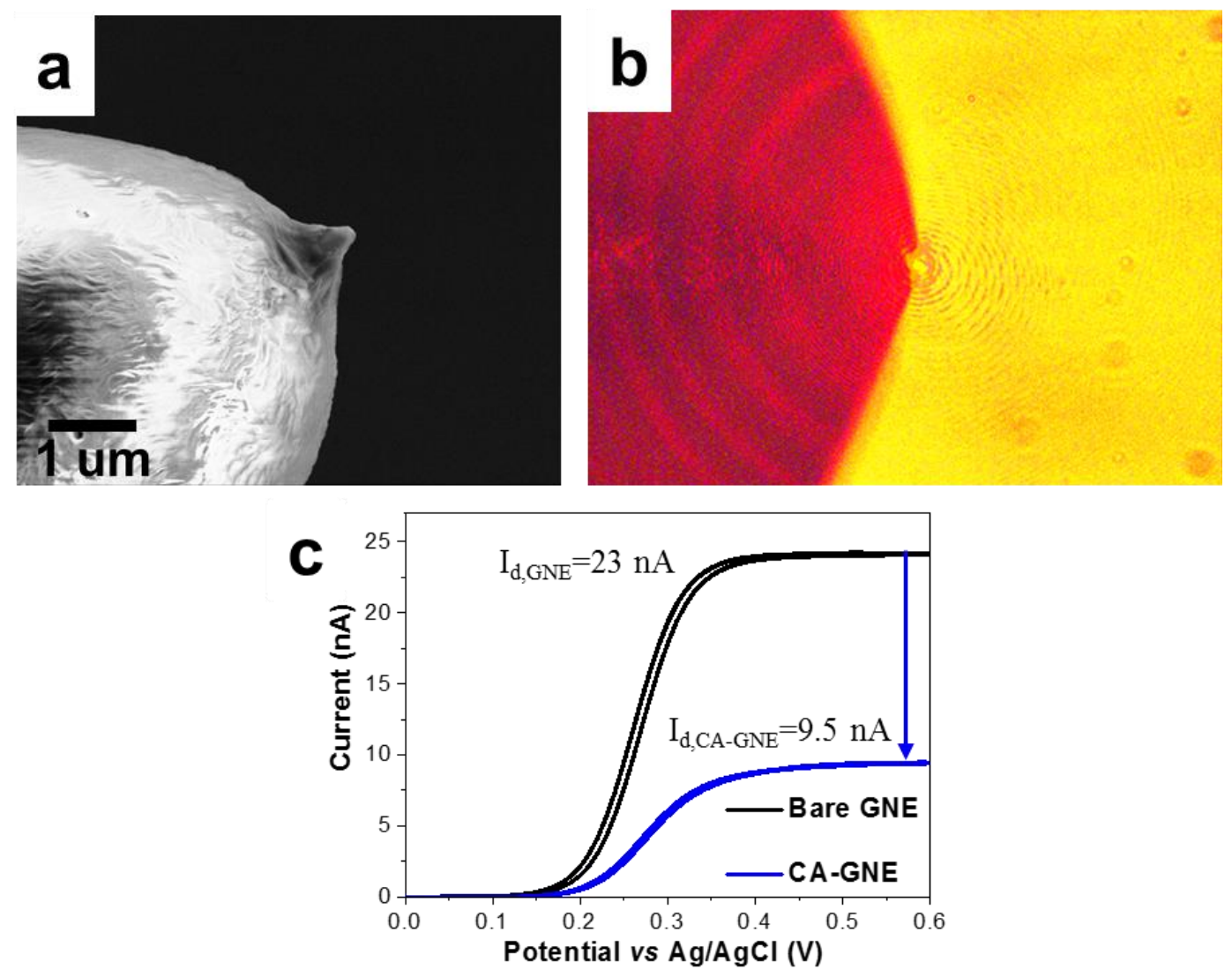

Fig. S1. a) SEM image of HDPE coated GNE; b) The optical microscope image of GNE with the laser beam focused on the apex; c) The CVs of GNE before (black) and after (blue) CA modification in $1 \mathrm{M} \mathrm{KCl}$ solution containing $100 \mathrm{mM}$ ferrocyanide ions. The sweep rate is $50 \mathrm{mV} / \mathrm{s}$. 


\section{The surface modification and characterization of gold nanoparticles}

$40 \mathrm{~nm}$ GNPs aqueous solution (150 pM) were purchased from Ted Pella, Inc. To form NHS ester terminated GNPs, $100 \mu \mathrm{L}$ of $5 \mathrm{mM}$ Lomant solution was added to $2 \mathrm{~mL}$ GNPs solution by 4 hours stirring. The mixture solution was further centrifuged to remove excess Lomant regents. The final product was adjusted to $2 \mathrm{~mL}$, the concentration of Lomant-GNPs was about $150 \mathrm{pM}$. Figure S2a shows the size distribution of Lomant-GNPs that was measured in DI water by a dynamic light scattering (DLS) method by Nano sizer (Nano-ZS, Malvern Instrument). The DLS data indicated that the average diameter of Lomant-GNPs was approximately $46 \mathrm{~nm}$. The GNPs before and after the Lomant functionalization were further examined in DI water by UV/vis spectrophotometry (CARY 300 Bio UV-Vis spectrometer). Figure S2b shows the UV-Vis extinction spectra of GNPs with a localized surface plasmon resonance peak at $530 \mathrm{~nm}$. A slightly red-shifted peak was observed for Lomant-GNPs, suggesting the successful surface modification of GNPs by Lomant reagent.

The Lomant-GNPs were also studied by Fourier-transform infrared spectroscopy (FTIR) (Tensor27, Bruker, Germany). The FTIR spectra shown in Figure S2c were taken from dried powder samples. The black colour spectrum of GNPs, which are capped with citrate, showed characteristic peaks of citrate. The broad peak at $1636 \mathrm{~cm}^{-1}$ corresponds to the $\mathrm{C}=\mathrm{O}$ symmetric stretching. The bands at $1390 \mathrm{~cm}^{-1}$ and $1033 \mathrm{~cm}^{-1}$ are assigned to $\mathrm{C}-\mathrm{H}$ stretching and $\mathrm{C}-\mathrm{O}$ stretching vibration modes, respectively. The blue spectrum of Lomant-GNPs in Figure S2c shows the characteristic IR peaks of Lomant reagents. The three noticeable IR peaks at $1088 \mathrm{~cm}^{-1}, 1202 \mathrm{~cm}^{-1}$ and $1741 \mathrm{~cm}^{-}$

1 are attributed to $\mathrm{C}-\mathrm{O}$ stretching mode, $\delta\left(\mathrm{CH}_{2}\right)$ scissoring vibration and $\mathrm{C}=\mathrm{O}$ symmetric stretching, respectively. These peaks are consistent with the IR peaks in the Lomant reagent powder, further confirming the successful modification of Lomant agent. 

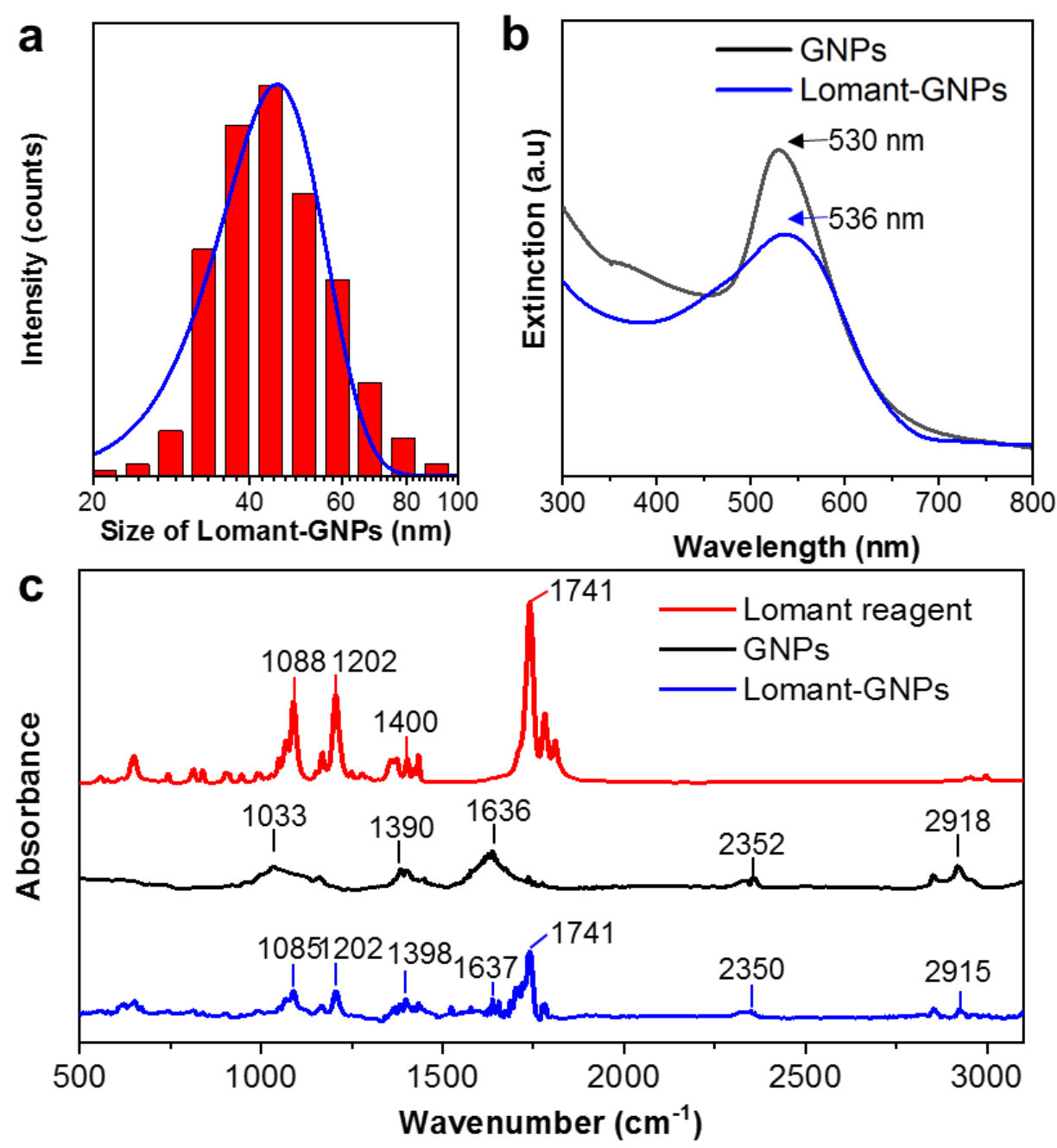

Fig. S2. a) Size distribution of Lomant-GNPs from DLS; b) UV-Vis extinction spectra for GNPs and Lomant-GNPs in DI water; c) FTIR spectra for Lomant reagents, GNPs and Lomant-GNPs in a dried powder.

\section{The experimental setup and data analysis}

The synchronization of SERS and electrochemical measurements was achieved by sending an external trigger signal (generated by a Labview program) to both CCD camera and Axon Digidata 1440A. A home-developed Matlab program was used to process the I-t data based on the previous reported methods. ${ }^{5-6}$ The current data was smoothed by using a 10 point moving average. The current baseline was flat and no further treatment was needed. The standard division $\sigma$ of the current baseline was calculated for every $100 \mathrm{~ms}$ trace section. The events were selected if the current change magnitude is higher than a threshold $(5 \sigma)$ above the baseline level. The characteristics (duration, amplitude, full width at half maxima, standard deviation) of the selected 
events were compared to be assigned to different types of events. The continually recorded SERS spectra by the CCD camera were also processed by the Matlab program. The time-resolved data were first cut to small sections for processing. The pixel number of the raw data was converted to wavelength (or wavenumber) based on the calibration file. The background of the SERS spectrum was fitted by the asymmetric least square method. For the heatmap, we only subtracted the average background of the first several frames. To correct the background effect of individual spectra, the background subtracted SERS spectra were further divided by the background accordingly. The Figure S3 a-c show the typical transient SERS spectra of three collision conditions (Types I, II and III), the corresponding background spectra and the background corrected spectra.

The laser power density was only approximately estimated in our experiments. We first placed a power meter near the GNE location at the microscope sample stage to measure the power (in $\mathrm{mW}$ ) of the laser beam. To measure the beam size, an image of the attenuated laser spot (with a $0.5 \mathrm{ND}$ filter) focused on a glass coverslip at the sample stage was captured via a CCD camera. The intensity profile across the laser spot image was fitted with a Gaussian function. The full width at half maximum of the Gaussian fit was used as the effective diameter of the laser beam to calculate the effective area of the laser spot by assuming a circular shape. The power density was then calculated as a ratio of the laser intensity $(\mathrm{mW})$ to the effective laser area $\left(\mu \mathrm{m}^{2}\right)$. 

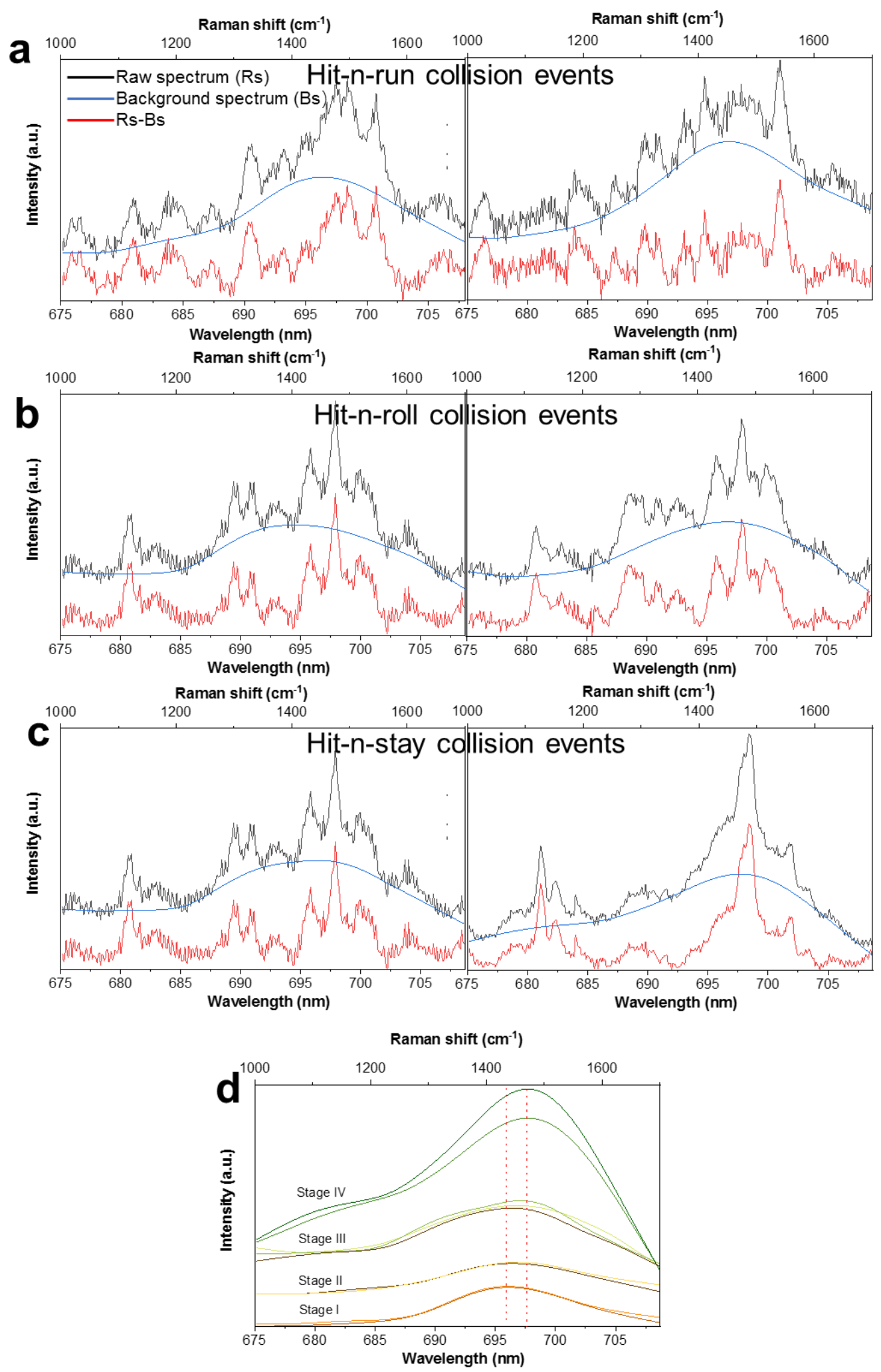

Fig. S3. (a-c) Typical SERS spectra (black) of three collision conditions (Type I, II and III), the corresponding background spectrum (blue) and the background corrected SERS spectra (red). d) The comparison of background spectra at different stages. We 
picked a few background spectra for each stage. The dash lines indicate the maxima of the background.

\section{The stability of vibration peaks during the whole experiments}
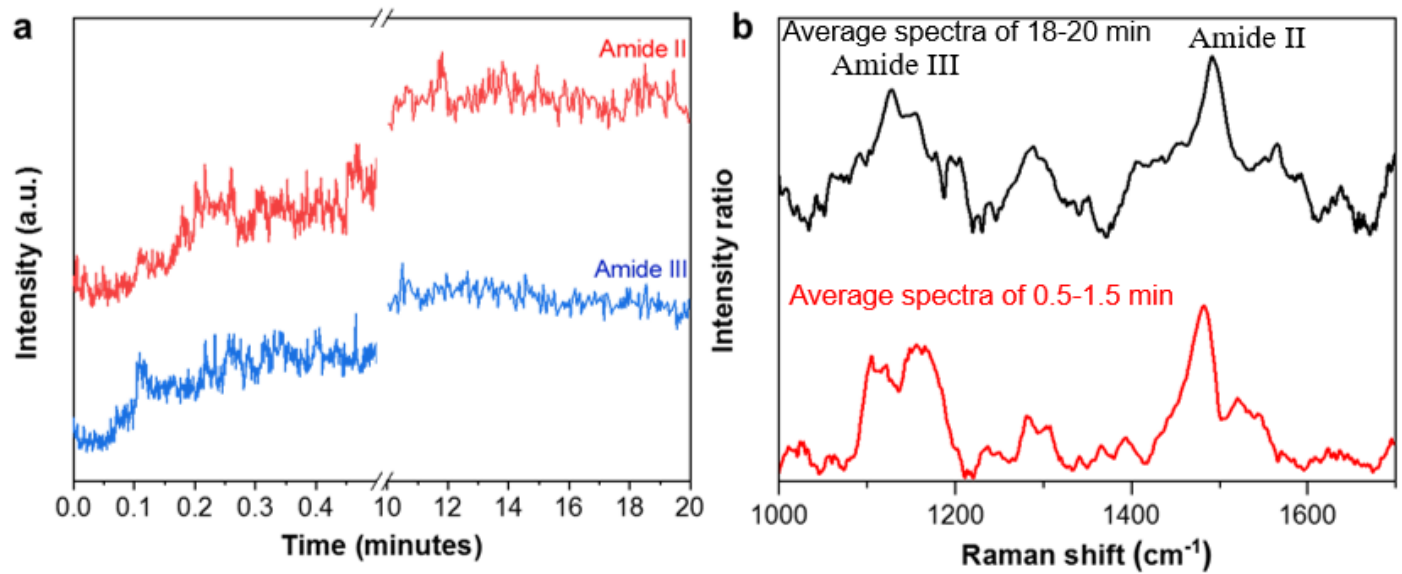

Fig. S4. a) The intensity time traces of two feature peaks: Amide II around $1500 \mathrm{~cm}^{-1}$ and Amide III near $1120 \mathrm{~cm}^{-1}$ during a 20 min measurement. b) The comparation of the averaged spectra (two-minutes time range) after background correction at the early (0.5$1.5 \mathrm{~min}$ ) and later time (last $2 \mathrm{~min}$ ) of the measurement.

\section{Control experiments and the assignment of SERS peaks}

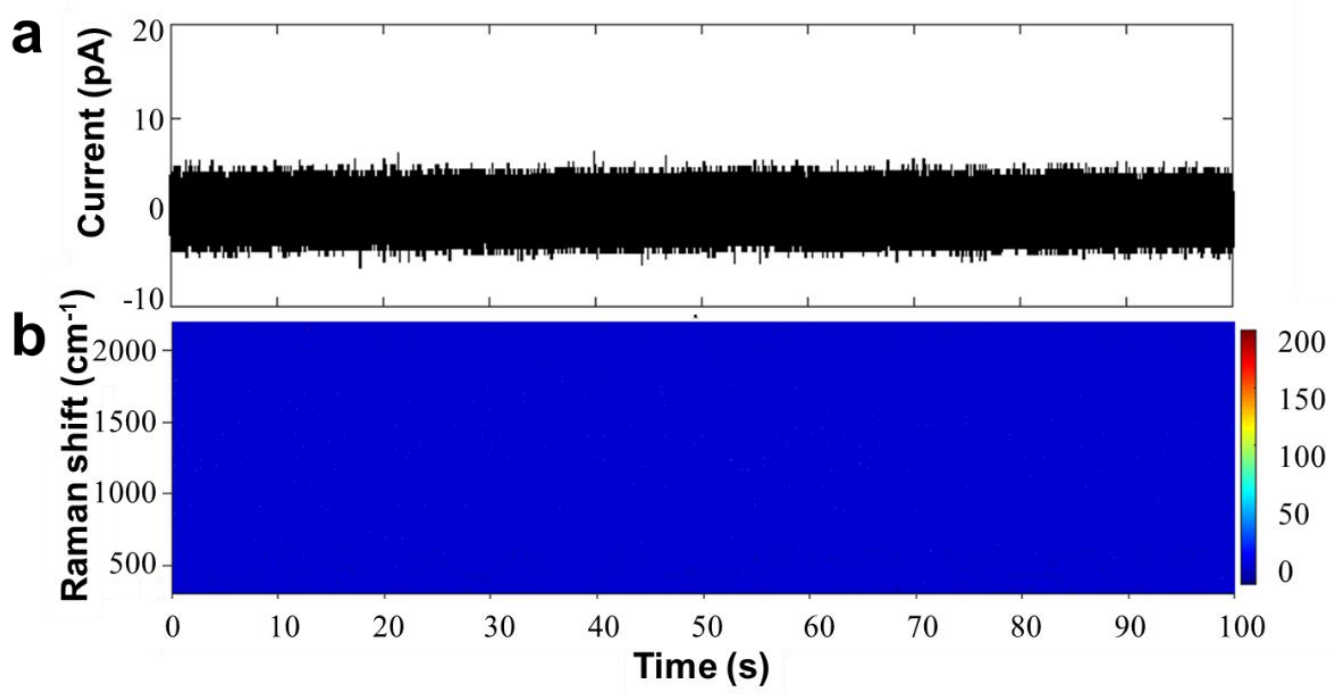

Fig. S5. a) Time-resolved EC current traces and b) SERS trajectory of a CA-GNE in 5 $\mathrm{mM}$ phosphate buffer with $3 \mathrm{mM}$ ferricyanide ions as redox probes in the absence of GNPs. The potential applied on the GNE was $600 \mathrm{mV}$ vs. $\mathrm{Ag} / \mathrm{AgCl}$ quasi reference electrode. 
To further understand the dynamic interaction, and likely reaction, between the NHS ester and the amine groups during and after the collision process, we designed the followed control experiments to investigate the molecular junctions formed from the collision. Firstly, no detectable current and Raman signal was observed with the CAGNE in the absence of GNPs as shown in Figure S5. Furthermore, the Raman spectra of cysteamine and Lomant reagent powder were also measured shown in Figure S6a to understand the molecular transformation in the formed NPoNE junction. Control experiments using (i) $20 \mathrm{pM}$ unfunctionalized GNPs on CA-GNE and (ii) LomantGNPs on bare GNE were conducted at the same condition in $5 \mathrm{mM}$ phosphate buffer (pH 7.4) with $3 \mathrm{mM}$ ferricyanide ions as the redox probes. The corresponding SERS spectra were collected as shown in Figure S6b. The SERS spectrum of GNP-CA-GNE structure exhibited seven main bands in the $1000-1700 \mathrm{~cm}^{-1}$ range. ${ }^{7-8}$ Compared with the normal Raman spectrum of CA powder, however, the intensity of SERS spectrum of GNP-CA-GNE was greatly reduced which could be negligible after Lomant-GNPs landing. In the spectra of Lomant, the peaks assignment is summarized in Table S1. The prominent band of $1488 \mathrm{~cm}^{-1}$ and $1608 \mathrm{~cm}^{-1}$ could be attributed to the $v(\mathrm{O}-\mathrm{N}-\mathrm{C})$ and $v(\mathrm{C}=\mathrm{O})$ from -OSu group. When new amide bonds are formed a new peak appears near $1471 \mathrm{~cm}^{-1}$ that is sharp and clearly resolved above the bump in the SERS spectrum. Meanwhile, the bands at $1608 \mathrm{~cm}^{-1}$ assigned to $\mathrm{v}(\mathrm{C}=\mathrm{O})$ from -OSu group disappeared with the release of -OSu group as shown in Fig.S6.
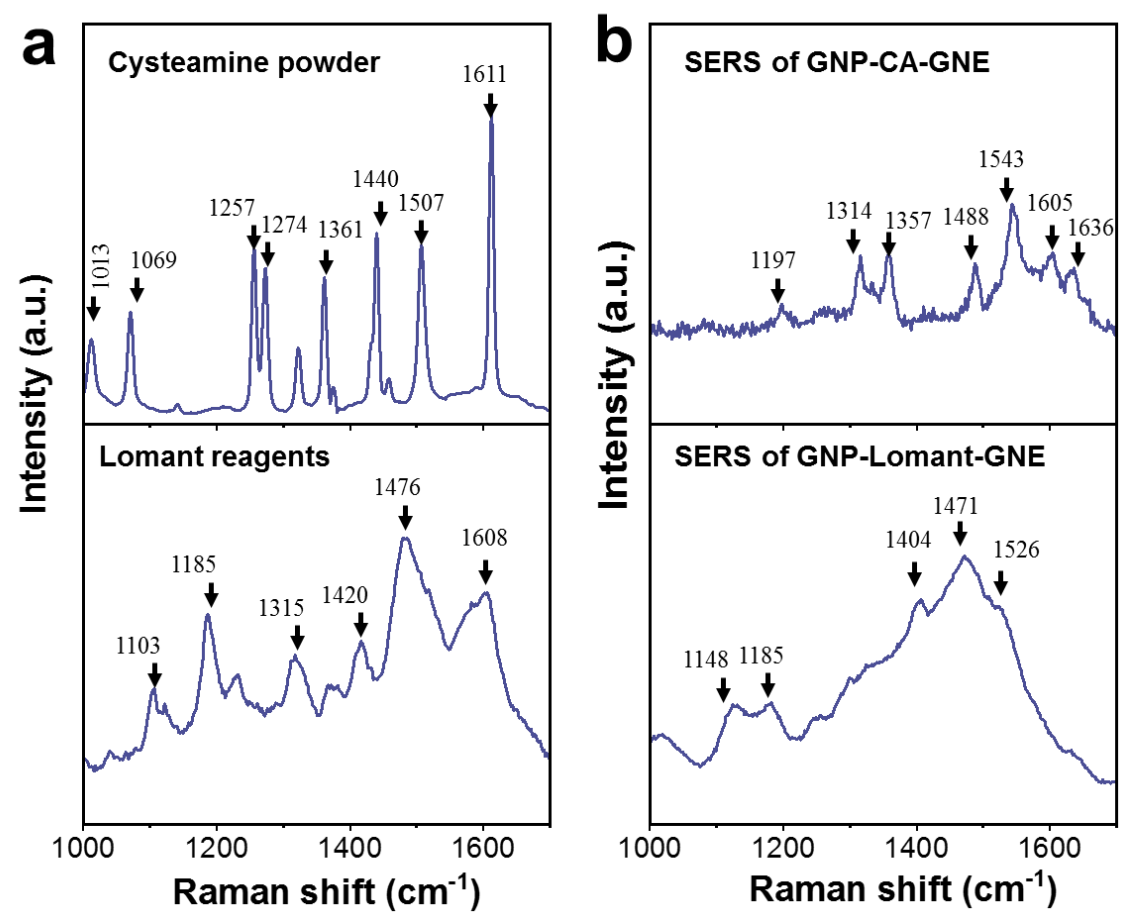
Fig. S6. a) Raman spectra of CA powder and Lomant reagents powder. b) SERS spectra of GNP-CA-GNE and GNP-Lomant-GNE structures.

Table S1. Raman peak assignments. ${ }^{7-9}$

\begin{tabular}{|c|c|c|c|c|c|}
\hline $\begin{array}{l}\text { Cysteamine } \\
\text { powder }\end{array}$ & $\begin{array}{l}\text { Lomant } \\
\text { reagent } \\
\text { powder }\end{array}$ & $\begin{array}{l}\text { SERS of } \\
\text { GNP- } \\
\text { CA- } \\
\text { GNE }\end{array}$ & $\begin{array}{l}\text { SERS of } \\
\text { GNP- } \\
\text { Lomant- } \\
\text { GNE }\end{array}$ & $\begin{array}{l}\text { Calculated } \\
\text { Amide } \\
\text { product }\end{array}$ & Assignments \\
\hline 1013 & & & & 1041 & $\begin{array}{l}v_{\mathrm{s}}(\mathrm{C}-\mathrm{C}-\mathrm{N}) \\
\text { wag }(\mathrm{NH})\end{array}$ \\
\hline \multirow[t]{3}{*}{1069} & & & & 1088 & $\begin{array}{l}v(\mathrm{C}-\mathrm{N}) \\
\mathrm{t}\left(\mathrm{CH}_{2}\right)_{\mathrm{S}}\end{array}$ \\
\hline & 1103 & & 1148 & & $\mathrm{v}(\mathrm{C}-\mathrm{N})$ \\
\hline & 1185 & 1197 & 1185 & 1225 & $\mathrm{v}\left(\mathrm{CH}_{2}-\mathrm{C}\right)$ \\
\hline 1257 & & & & 1245 & $\mathrm{t}\left(\mathrm{CH}_{2}\right)_{\mathrm{N}}+\mathrm{t}(\mathrm{NH})$ \\
\hline \multirow[t]{2}{*}{1274} & & & & 1271 & $\mathrm{t}\left(\mathrm{CH}_{2}\right) \mathrm{s}$ \\
\hline & 1315 & 1314 & & 1330 & $\mathrm{t}\left(\mathrm{CH}_{2}\right)_{\mathrm{N}}$ \\
\hline 1361 & & 1357 & & 1362 & $\operatorname{wag}\left(\mathrm{CH}_{2}\right)_{\mathrm{N}}$ \\
\hline \multirow[t]{2}{*}{1440} & 1420 & & 1404 & 1440 & $\delta\left(\mathrm{CH}_{2}\right) \mathrm{s}$ \\
\hline & 1476 & 1488 & 1471 & 1465 & $\mathrm{v}(\mathrm{C}-\mathrm{N})$ \\
\hline \multirow[t]{2}{*}{1507} & & 1543 & 1526 & 1471 & $\delta\left(\mathrm{CH}_{2}\right)_{\mathrm{N}}$ \\
\hline & 1608 & & & 1722 & $\mathrm{v}(\mathrm{C}=\mathrm{O})$ \\
\hline 1611 & & 1636 & & & $\delta\left(\mathrm{NH}_{2}\right)$ \\
\hline
\end{tabular}


Table S2 Raman peak assignment of amide bonds.

\begin{tabular}{lllll}
\hline Mode description & Mode assignment & $\begin{array}{l}\text { Peak } \\
\text { observed } \\
\left(\mathbf{c m}^{-\mathbf{1}}\right)\end{array}$ & $\begin{array}{l}\text { Peak } \\
\text { calculated } \\
\left(\mathbf{c m}^{-\mathbf{1}}\right)\end{array}$ & $\begin{array}{l}\text { Ref }^{\mathbf{1 0}-} \\
\mathbf{1 1}\end{array}$ \\
\hline Amide I & $v(\mathrm{C}=\mathrm{O})$ & $\begin{array}{l}\text { Not } \\
\text { detectable }\end{array}$ & 1804 & 1665 \\
& & $1480 / 1500$ & 1463 & 1556 \\
Amide II & $v(\mathrm{C}-\mathrm{N})$ & $1100-1200$ & $1100-1300$ & 1265 \\
\hline
\end{tabular}
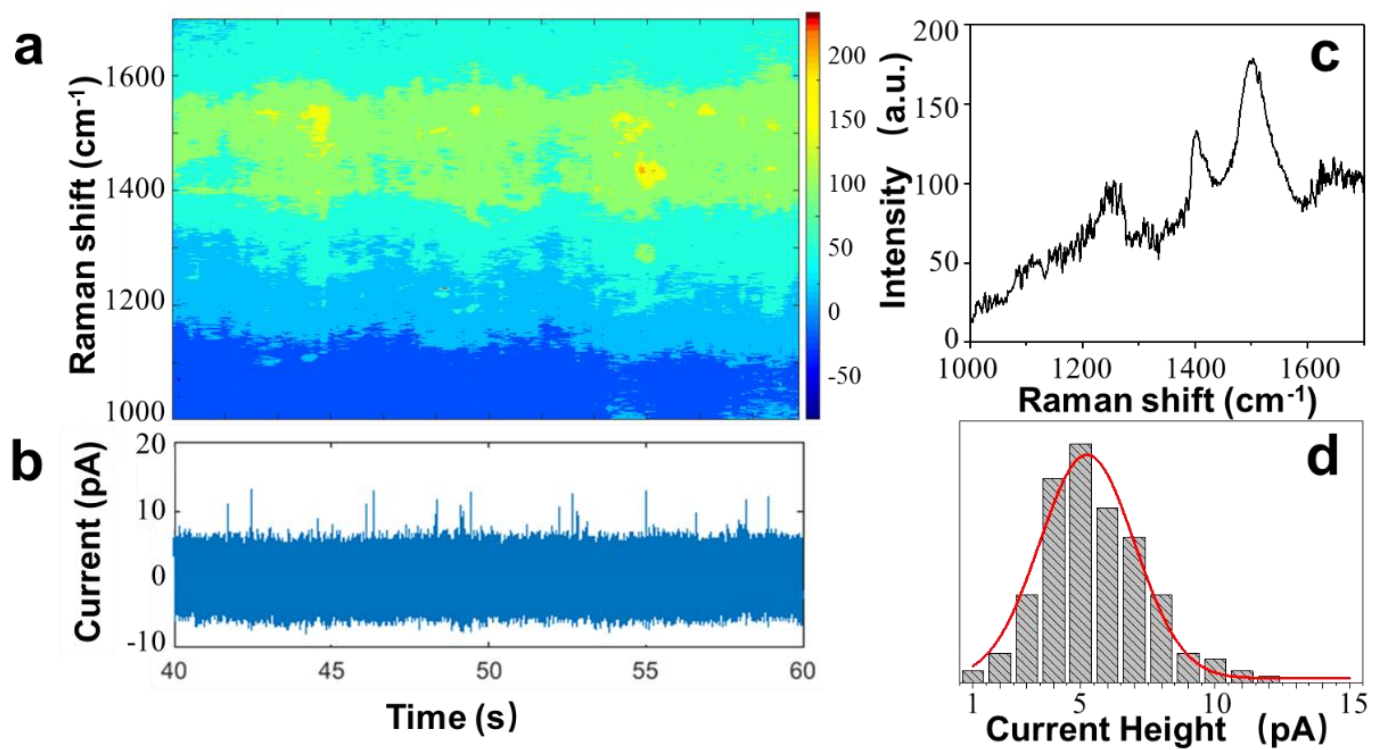

Fig. S7. a-b) Time-resolved SERS trajectory(a) and EC current-time (I-t) trace (b) collected from a 3-MPA-GNE after adding $20 \mathrm{pM}$ Lomant-GNPs in the solution of 5 $\mathrm{mM}$ phosphate buffer containing $3 \mathrm{mM}$ ferricyanide. c) Typical average Raman spectrum from a. d) Current peak height distribution. 
a
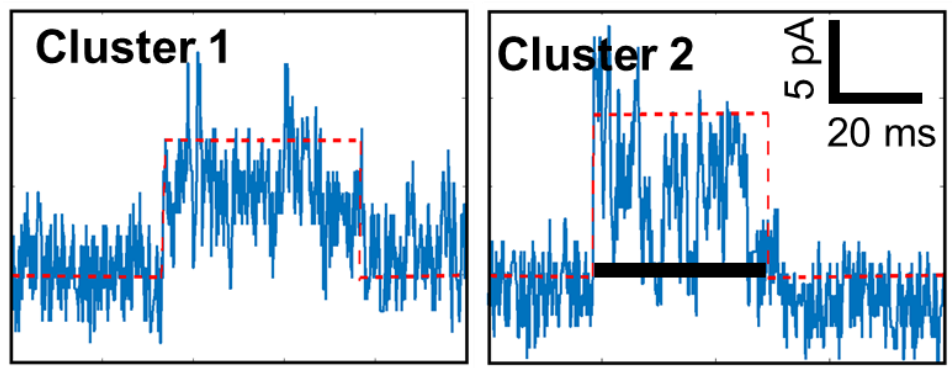

b

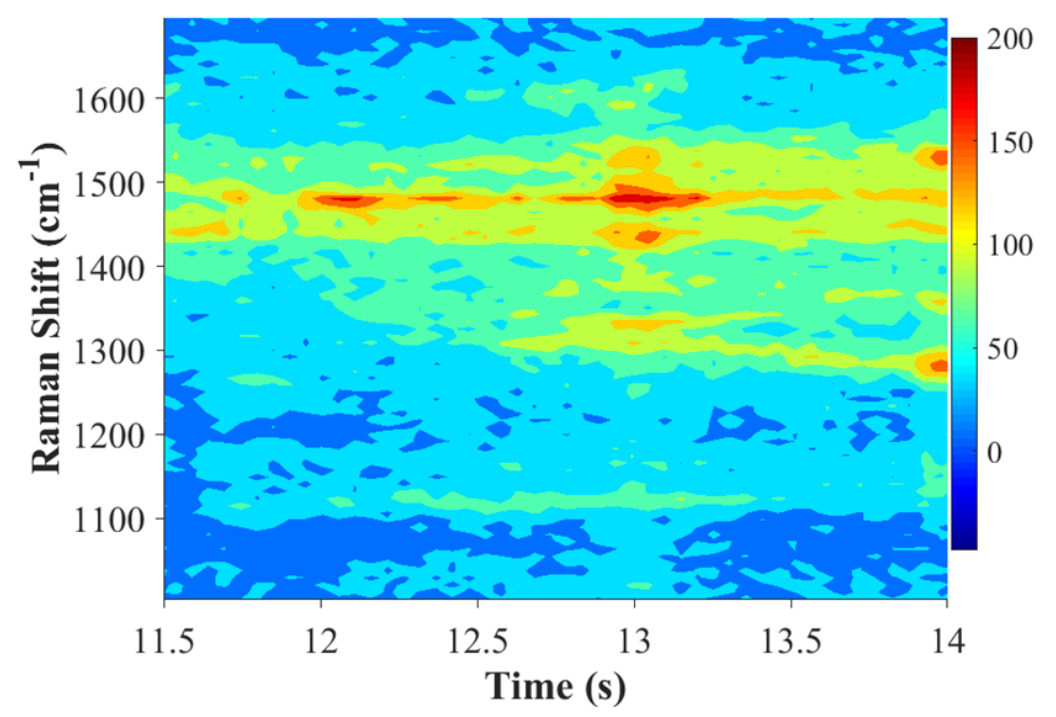

Fig. S8. a) Zoom-in view of two EC current cluster signals and b) Zoom-in view of the SERS heatmap shown in Fig.5.
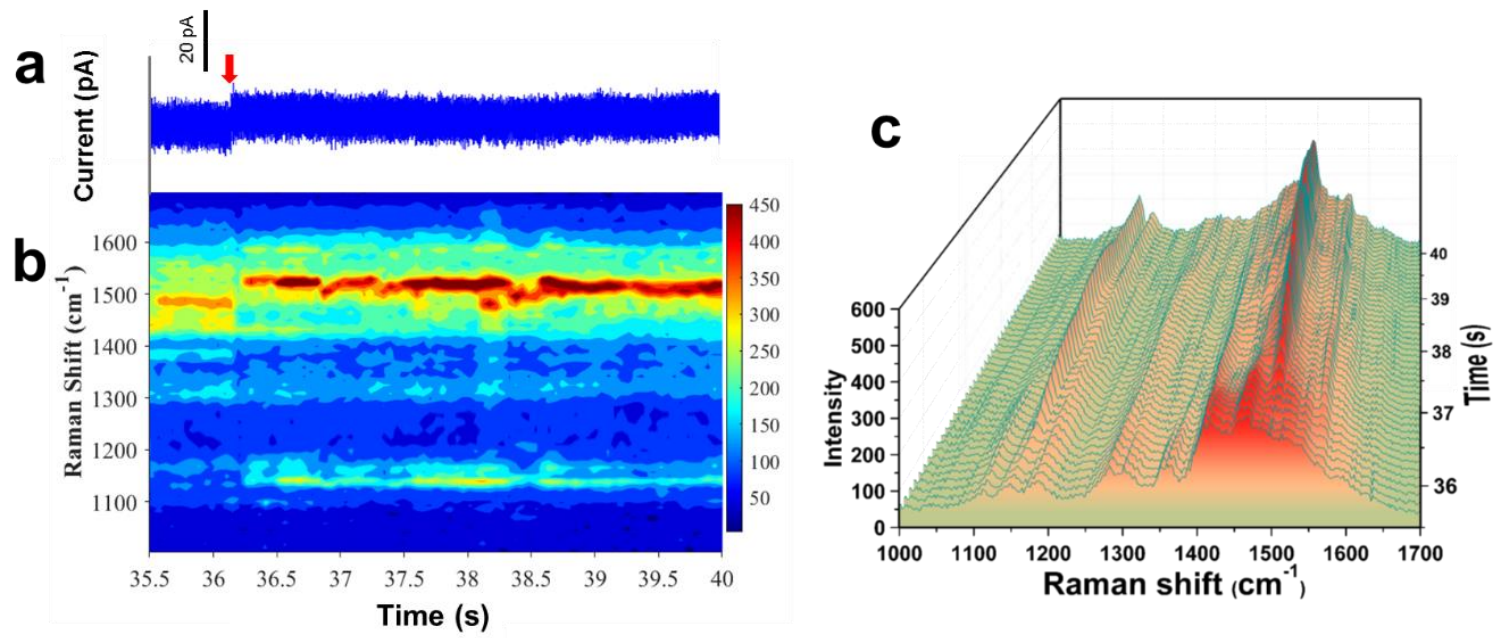

Fig. S9. Typical time-resolved SERS trajectories induced by 'hit-n-stay' collision events of Lomant-GNPs. 


\section{Raman spectra calculations by Density Functional Theory (DFT)}

The geometry optimization and Raman spectra calculation were conducted by using DFT methods using functional B3LYP. The basis sets are $6-311+\mathrm{G}^{* *}$ for carbon, nitrogen, hydrogen, oxygen and sulphur atoms and LANL2DZ for gold atoms. ${ }^{12}$ Calculations were carried out with the Gaussian 09 program package. ${ }^{13}$ A scaling factor of 0.963 was used for all the vibrational bands.

\section{References:}

(1) Ren, B.; Picardi, G.; Pettinger, B. Preparation of gold tips suitable for tip-enhanced Raman spectroscopy and light emission by electrochemical etching. Rev. Sci. Instrum. 2004, 75, 837841.

(2) Tuchband, M.; He, J.; Huang, S.; Lindsay, S. Insulated gold scanning tunneling microscopy probes for recognition tunneling in an aqueous environment. Rev. Sci. Instrum. 2012, 83, 015102.

(3) Zoski, C. G. Steady-state voltammetry at microelectrodes. 1996.

(4) Wirde, M.; Gelius, U.; Nyholm, L. Self-Assembled Monolayers of Cystamine and Cysteamine on Gold Studied by XPS and Voltammetry. Langmuir 1999, 15, 6370-6378.

(5) Plesa, C.; Dekker, C. Data analysis methods for solid-state nanopores. Nanotechnology 2015, 26, 084003.

(6) Chang, S.; Huang, S.; He, J.; Liang, F.; Zhang, P.; Li, S.; Chen, X.; Sankey, O.; Lindsay, S. Electronic Signatures of all Four DNA Nucleosides in a Tunneling Gap. Nano Lett. 2010, 10, 1070-1075.

(7) Riauba, L.; Niaura, G.; Eicher-Lorka, O.; Butkus, E. A Study of Cysteamine Ionization in Solution by Raman Spectroscopy and Theoretical Modeling. J. Phys. Chem. A 2007, 110, 13394-13404.

(8) Zhang, D.; Si, M.; Kang, Y.; Liu, Z.; Liu, R. The Study on Vibrational Raman Spectrum and SERS Spectra on Sliver film of Cysteamine Molecule. J. Light Scattering 2010, 022, 4448.

(9) Jiang, X.; Yang, M.; Meng, Y.; Jiang, W.; Zhan, J. Cysteamine-Modified Silver Nanoparticle Aggregates for Quantitative SERS Sensing of Pentachlorophenol with a Portable Raman Spectrometer. ACS Appl. Mater. Interfaces 2013, 5, 6902-6908.

(10) Kurouski, D.; Postiglione, T.; Deckert-Gaudig, T.; Deckert, V.; Lednev, I. K. Amide I vibrational mode suppression in surface (SERS) and tip (TERS) enhanced Raman spectra of protein specimens. Analyst 2013, 138, 1665-1673.

(11) Lim, C. Y.; Owens, N. A.; Wampler, R. D.; Ying, Y.; Granger, J. H.; Porter, M. D.; Takahashi, M.; Shimazu, K. Succinimidyl Ester Surface Chemistry: Implications of the Competition between Aminolysis and Hydrolysis on Covalent Protein Immobilization. Langmuir 2014, 30, 12868-12878.

(12) Wu, D.-Y.; Liu, X.-M.; Duan, S.; Xu, X.; Ren, B.; Lin, S.-H.; Tian, Z.-Q. Chemical Enhancement Effects in SERS Spectra: A Quantum Chemical Study of Pyridine Interacting with Copper, Silver, Gold and Platinum Metals. J. Phys. Chem. C 2008, 112, 4195-4204.

(13) Frisch, M.; Trucks, G.; Schlegel, H.; Scuseria, G.; Robb, M.; Cheeseman, J.; Scalmani, G.; Barone, V.; Mennucci, B.; Petersson, G. Gaussian 09 package. Pittsburgh PA: Gaussian Inc 2009. 